\title{
Growth retardation-mild developmental delay-chronic hepatitis syndrome
}

INSERM

\section{Source}

INSERM. (1999). Orphanet: an online rare disease and orphan drug data base. Growth retardation-mild developmental delay-chronic hepatitis syndrome. ORPHA:391366

Growth retardation-mild developmental delay-chronic hepatitis syndrome is a rare, genetic, parenchymatous liver disease characterized by pre- and postnatal growth retardation, mild global developmental delay, chronic hepatitis with hepatosplenomegaly, Hashimoto thyroiditis, thrombocytopenia, anemia, and B-precursor acute lymphoblastic leukemia. 